\title{
El Estigma en la Representación Social de la Lepra ${ }^{1}$
}

\author{
The Stigma in the Social Representation of Leprosy
}

\author{
Alexis Romero-Salazar'; María Cristina Parra²; Carmen Moya-Hernández²; \\ Raima Rujano \& Johel Salas ${ }^{2}$
}

ROMERO-SALAZAR, A.; PARRA, M. C.; MOYA-HERNÁNDEZ. C. M.; RUJANO, R. \& SALAS, J. The Stigma in the Social Representation of Leprosy. Cad. Saúde Públ., Rio de Janeiro, 11 (4): 535-542, Oct/Dec, 1995.

Social representation of leprosy in active patients under medical control is made up elements that come from the medical discourse and also from the discourse that about this disease circulates in society. This syncretism is expressed in each context a specific way on the components of the representation notion, beliefs, attitudes and behaviors - making of it a complex reality.

On account of beliefs importance, whose depth and roots are linked to the thousand of years this disease has been known and to the seclusion practices in force till recent times, it would be comprehensible the predominance of elements related to common sense or popular knowledge. Nevertheless, as the representation is a collective construction, its fundamental features would be defined according to the specific characteristics of the group. To this respect, the main finding of this research referred to: first, the limited knowledge around this disease and the existence of beliefs and inadequated attitudes for controlling programs of active patients registered in the Maracaibo Service of Sanitary Dermatology located in Venezuela (Servicio de Dermatologia Sanitaria de Maracaibo (SDSMJ, Venezuela); second, a predominance of a congnitive dimension of representation, notions and beliefs relate to the role of fortune and fate, sexual promiscuity or heritage in the contagion; third, a patient's tendency to reproduce a disqualified stereotype and hide the disease due to an exaggerated rejection fear.

Key words: Leprosy; Social Representation; Stigma

\section{INTRODUCCION}

No obstante el progreso del conocimiento en torno a los aspectos biomédicos de la lepra, incluida la terapéutica, sería aventurado hablar de control definitivo de la enfermedad. Ello en tanto los afectados no sólo siguen sometidos al riesgo de las deformidades que los incapacitan para el

\footnotetext{
${ }^{\prime}$ Esta investigación fue auspiciada por el Programa de Pequeñas Becas para Investigaciones en Aspectos Sociales y Económicos de las Enfermedades Tropicales del Laboratorio de Ciencias Sociales-Universidad Central de Venezuela, con apoyo financiero del Programa Especial de Investigación y Entrenamiento en Enfermedades Tropicales. UNDP/BANCO MUNDIAL/OMS.

${ }^{2}$ Universidad del Zulia. Av. Universidad, № 25-266, Maracaibo, Venezuela.
}

trabajo, sino también a la inhabilitación social que deriva del rechazo de que son objetos (Fassin, 1990; de Rojas, 1989). En tales condiciones se hace difícil, primero, que los enfermos asuman su afección como cualquier otra, susceptible de ser curada y, segundo, que puedan llevar una vida social y familiar armónica.

Este problema podría guardar relación con una representación social de la lepra que se ha sustentado en arraigados prejuicios, y antiguas prácticas confinatorias que fueron corrientes hasta épocas recientes (Kauffman, 1985). Porque los elementos constitutivos de esta representación se generan en el marco en el que se produce también el proceso general de inhabilitación social de las personas que portan la enfermedad.

El estudio se propuso explorar, a través del mismo enfermo, las nociones, las creencias y 
las actitudes que giran en torno a la lepra y que obviamente tienen para él una significación vital, en tanto podrían movilizar y condicionar su comportamiento ante la afección. Con ello se intentó revisar los contenidos de la experiencia y los elementos de significación característicos del contexto socio-cultural, que como tales son claves para explicar y comprender el comportamiento social de los individuos frente a la enfermedad.

En atención a ello se plantearon las siguientes interrogantes: ¿Cuáles son las ideas que el paciente de lepra tiene de su enfermedad? ¿Está vinculada la inhabilitación social a la representación de la afección? La formulación de tales preguntas estuvo vinculada a la consideración de que las nociones y las creencias del leproso podrían determinar su comportamiento frente al tratamiento, facilitándolo a obstaculizándolo (Claro, 1995).

A tal efecto se diseñó una investigación de carácter descriptivo-exploratorio, que estableció como objetivos generales: analizar la representación que tiene el leproso de su enfermedad, así como analizar el manejo de la información y la búsqueda de opciones, por parte de los enfermos de lepra, para superar su estigma. La identificación de las nociones, actitudes y creencias podría potenciar la posibilidad de planificar acciones encaminadas a incidir en aquellos aspectos cognoscitivos, afectivos y conductuales que obstaculizan el tratamiento y el relacionamiento social del enfermo.

\section{CONSIDERACIONESTEORICAS}

\section{La lepra se refiere a un atributo} profundamente desacreditador; es decir, estigmatiza a la persona afectada y el grado de estigmatización se expresa en la caracterización que, social a históricamente, se ha hecho de la enfermedad.

Desde una perspectiva sociológica, interesa analizar el estigma en relación a la "Carrera Moral”, la cual está representada por todas las experiencias de aprendizaje por las que pasa la persona con lepra una vez declarada su enfermedad, haciéndole conocer y aprehender su condición - se hace consciente de que es diferente al resto de los normales, aprende que es portadora de un estigma y capta las consecuencias de ello (Goffman, 1961, 1970).

Así, en la medida que el estigma sea invisible y conocido sólo por el individuo que to posee, será más fácil mantener el secreto ante los demás. En algunos casos, puede decidir qué información va a ocultar sobre su persona y es a sus allegados a quienes puede querer ocultar con más celo su afección. Esto es to que Goffman llama "encubrimiento".

Otra situación es la del "enmascaramiento": ya no se trata de manejar información con el fin de ocultar, sino con el objetivo de encubrir el atributo; tal es el caso de aquellos individuos que, por to general, cambian el nombre de su enfermedad; dicen hongos en vez de lepra, por ejemplo. Esto to hacen básicamente con aquellas personas que desconocen la afección, o están fuera de su núcleo familiar. El individuo entonces viste con ropa adecuada para ocultar señales, manchas y deformidades, típicas de la lepra.

Pero las conductas encubridoras y/o enmascaradoras se concretan con arreglo a las condiciones de los individuos afectados: aquellos cuyo estigma es visible y conocido por los demás - manchas en la piel, parálisis (desacreditados) - tienen la necesidad de manejar la tensión resultante de sus encuentros con los otros. Aquellos que tienen su estigma que no es conocido ni inmediatamente perceptible - enfermos de lepra con bajo grado de incapacidad física, sin machas ni deformidades muy visibles (desacreditables) - tienen necesidad de controlar, en el medio en que se desenvuelven, la información sobre su condición de estigmatizados (Hasselblad, 1975). El estigma en lepra, entonces, puede ser asumido como un proceso mediante el cual se restringe el papel social del individuo a partir del momento del diagnóstico. Tal proceso, que provocaba, hace pocos años, una segregación compulsiva, aún hoy continúa representando la muerte social y el ingreso del individuo a una categoría no humana. Por otro lado, la restricción supone la consideración del enfermo como culpable de su afección (Valencia, 1989). 
Siendo que las representaciones son figuraciones mentales aprendidas por vía de la socialización - introduce valores y reglas de conducta -, cada sociedad selecciona los atributos que constituirán el concepto de normalidad, y, por oposición, define la noción de desvío. Así, cualquier elemento que no coincida con las definiciones culturalmente postuladas, se convierte en un peligro. Tal es el caso de algunas enfermedades como la lepra que, en su visión estereotipada, causa deformidades en el cuerpo; porque, siendo éste una representación de la sociedad, no sólo como proceso exclusivamente biológico, sus significantes en el plano fisiológico vienen a representar fenómenos sociológicos (Rodríguez, 1983).

\section{METODOLOGIA}

La relación entre to cualitativo y to cuantitativo no puede ser pensada en términos de oposición contradictoria. Porque "las relaciones sociales pueden ser analizadas en sus aspectos más 'ecológicos' y 'concretos' y profundizadas en sus significados más esenciales. Así, el estudio cuantitativo puede generar temas para ser profundizados cualitativamente y viceversa" (Sanches \& Minayo, 1993: 247).

Entonces, como se trata de producir conocimiento, queda justificada la utilización complementaria de los métodos y técnicas que en ambos abordajes se han generado (Romero Salazar, 1990). En el caso de esta investigación, se produjo un doble movimiento cuanti-cualitativo, dirigido, en primer lugar, a aportar datos, indicadores y regularidades observables en las nociones y creencias, actitudes y comportamientos en torno a la lepra y, en segundo lugar, a profundizar en la complejidad del fenómeno. En el primer caso se trataba de un estudio extensivo, en el segundo de uno intensivo.

La técnica de la encuesta fue utilizada para describir la regularidad - más en la superficie en torno a to que saben y piensan los leprosos de su afección. La técnica del Relato de Vidaen este caso de enfermedad - fue la empleada para aprehender elementos anclados más profundamente en la subjetividad de los leprosos, en to que tiene que ver con to que sienten y hacen gnte su afección.

Se diseñaron así dos instrumentos de naturaleza distinta: el cuestionario y la entrevista a profundidad.

La población estuvo conformada por 774 individuos con la enfermedad de lepra, que recibían tratamiento ambulatorio (activos) en el Servicio de Dermatología Sanitaria del Estado Zulia (Venezuela), durante el primer semestre de 1994. De ellos se seleccionó probabilísticamente por estratificación por afijación proporcional - una muestra de 40 pacientes, distribuídos por estratos, (de acuerdo con el grado de incapacidad) para la aplicación del cuestionario. Para la entrevista a profundidad se seleccionaron intencionalmente nueve enfermos de ambos sexos y distintos grados de incapacidad.

\section{ANALISIS}

La información proveniente del cuestionario fue procesada mediante el use del paquete SAS. En un primer momento se practicó un análisis porcentual a partir de las tablas de distribución de frecuencias. En un segundo momento, se calcularon los indices de ostracismo, de ocultamiento, de estereotipización, de miedo al rechazo, de incapacitación y de fatalismo. Los resultados obtenidos fueron integrados para producir el coeficiente de estigmatización.

El análisis cualitativo se inició a partir de la transcripción de los relatos. Para la interpretación fue necesaria una adaptación del procedimiento propuesto por Poirier, Clapier-Valledon y Raibaut (Poirier et al., 1983).

En la primera fase se organizaron y ordenaron los relatos, a partir de la revisión de la transcripción y comparación con la grabación y codificación de los documentos.

En la segunda fase se produjo la clarificación del Corpus del relato a través del establecimiento del perfil biográfico y del léxico-repertorio de cada uno de los entrevistados. Para esto último se afinaron los campos semánticos (categorización). 
En la tercera fase se integraron los sinónimos y expresiones utilizados por los entrevistados y que aparecen en la ficha individual.

En la cuarta fase se realizó el inventario de significaciones, es decir, se organizaron los significados en categorías, mediante la utilización de la llamada "rejilla de análisis".

\section{RESULTADOS}

Tratándose de un doble movimiento metodológico, que permitió la captación de las nociones, las creencias, las actitudes y los comportamientos implícitos en la representación social, los resultados se presentan en dos panes, para producir luego su articulación a nivel de las conclusiones.

\section{Primero Nivel de Análisis. Los Porcentajes}

\section{La Percepción de Reacción Social Negativa}

En general, las respuestas de los pacientes tienden a negar la existencia de claras actitudes de rechazo por parte de los familiares más cercanos. Como manifestación de rechazo sólo señalan el que apartaran sus prendas de vestir y otras cosas de use personal.

Específicamente en to que tiene que ver con la relación de pareja (en el caso de quienes la tenían o la tienen), los enfermos no perciben que hayan sido o sean discriminados.

Tal vez por ello, $92,5 \%$ expresan que se sienten muy cerca de su familia y $90 \%$ señalan que se sienten protegidos por ella. Esa percepción podría haberse construido a partir de reales situaciones no discriminatorias, en tanto las condiciones predominantes en estos enfermos están referidas a la casi total inexistencia de deformidades (en su mayoría incapacidad 0).

En relación al plano de la vida comunitaria, $60 \%$ de los pacientes cuyos vecinos están enterados de su afección dicen haber sido objeto de la reacción social negativa, no tanto porque las manifestaciones de la enfermedades sean visibles, sino por la idea que tienen de la lepra.
Igualmente, en el plano laboral, en el caso de los patrones que fueron enterados del padecimiento del trabajador, la percepción de los enfermos es que existe algún rechazo (el $30 \%$ de ellos fueron despedidos). En relación al sindicato, la percepción es que, de estar la Junta Directiva en conocimiento de la enfermedad del trabajador, no habría solidaridad; por esto se cuidan de no enterarla (sólo el 7\% de los pacientes to hacen).

\section{La Auto-identificación}

En cuanto a la caracterización física de la persona enferma, se tiene que el $80 \%$ de los pacientes encuestados la definen como débil, con manchas y deformidades. Esa calificación negativa se ve reforzada con el señalamiento de la introversión (45\%), el mal humor y la agresividad (30\%) como rasgos conductuales predominantes en los leprosos. Desde el punto de vista socioeconómico, las características que le asocian son la pobreza, la procedencia rural y la falta de educación.

\section{Miedos y Temores}

Los enfermos encuestados manifiestan temor a contagiar a su familia $(45 \%)$ y a otras personas con las que tengan contacto $(42 \%)$. Igualmente tienen miedo a que, conociendo su afección, los otros los rechacen (62.5\%), así como a que los expulsen de sitios públicos $(65 \%)$.

\section{Ocultamiento}

En opinión del 85\% de los pacientes encuestados, los enfermos ocultan la afección (60\%, siempre, y $25 \%$, con mucha frecuencia). Las razones que tendrían para hacerlo las vinculan al temor a ser rechazados por los amigos (80\%) y a ser despedidos del trabajo $(30 \%)$ y a ser estereotipados como anormales (85\%). Estos resultados contrastan con el $25 \%$ que aceptan haber enmascarado y encubierto su afección, bien tapando las manchas o lesiones, acudiendo a escondidas a la Unidad de Dermatología Sanitaria, o bien dándole otro nombre a la enfermedad o negando la denominación de los medicamentos. 


\section{Percepción de Cambios de la Primera Fase de la Enfermedad}

En to que se retiere a los hábitos cotidianos, la mayoría de los pacientes no recuerda que se hayan producido cambios. No obstante, es importance señalar que, en el plano de la personalidad, reconocen modificaciones al momento de enterarse que tenían lepra: $60 \%$ dicen que se alteraron los nervios, $37 \%$ se sintieran "anormales" y otros con mal olor.

\section{Segundo Nivel de Análisis: El Coeficiente de Estigmatización}

Los resultados presentados en términos porcentuales nos colocaron en posición de producir una estimación - cuantitativa - de la profundidad de las nociones y creencias que fueron investigadas a través del cuestionario.

En relación a ello se realizó el cálculo de varios índices:

- Indice de Ocultamiento: alcanza un $74.8 \%$ que revela la existencia de un elevado nivel de encubrimiento y enmascaramiento. Siendo irrelevantes las diferencias por grado de incapacidad. En rigor, los enfermos, por el temor al rechazo, ocultan su afección la mayoría de las veces y cualquiera sea la circunstancia. Hay que asumir que la necesidad de encubrir retrae a los individuos, generándoles problemas para su integración en los diferentes planos de la vida social.

- Indice de Ostracismo: con un $23.6 \%$ (no hay diferencias por grado de incapacidad), pone en evidencia la naturaleza contradictoria del comportamiento de los enfermos, en tanto el bajo nivel de este índice contrasta con el $74.8 \%$ de ocultamiento; to cual podría ser explicado de que, logrando ocultar su afección, no tienen porque aislarse o esconderse de los otros considerados normales.

Encubriendo y enmascarando se protegen del rechazo.

De todas maneras, habría que enfatizar la inconsistencia de las respuestas al respecto, tal vez derivada de la resistencia a aceptar la autoexclusión.

- Indice de Estereotipización: alcanzó un $68.1 \%$ (irrelevantes las diferencias por grado de incapacidad), evidenciando una significativa tendencia a la autoidentiticación negativa, que constituye un mecanismo fundamental de la estigmatización. En la medida en que el estereotipo corresponde a un individuo desacreditado, el paciente percibirá mayores barreras, inhabilitándose para llevar una vida social y familiar armónica.

- Indice de miedo al Rechazo: que llega al 100\% (diferencias irrelevantes), ratificando resultados de trabajos anteriores que definen al leproso como un sujeto lleno de miedos.

Estos índices fueron integrados y ponderados, según su fuerza predictiva, para producir el Coeficiente de Estigmatización, que alcanzó un $67 \%$ indicativo de una subjetividad (expresada en esta parte sólo en términos de nociones y creencias) que obstaculiza la integración o reinserción social y productiva del enfermo.

Es importante señalar la inconsistencia de las diferencias entre las nociones y creencias de los individuos con incapacidades distintas. Ello confirma nuestra intuición en torno a la homogeneidad de los elementos constitutivos de la representación social del enfermo. En otro plano, el de las actitudes y conductas, se establece, una independencia de éstas y las lesiones o deformidades que se posean. Esta uniformidad del comportamiento tendria que ver con una representación social que iguala a todos los enfermos; cualquiera sean sus características, con deformidades o sin ellas, todos son leprosos, y eso es to que importa en la situación de estigma. 


\section{DATOS DE LA ENTREVISTA A PROFUNDIDAD (RELATOS)}

\section{Actitudes y Conductas Estigmatizadas}

\section{Ocultamiento}

La máxima expresión del estigma es el ocultamiento en sus dos acepciones (enmascaramiento y encubrimiento), al respecto se tiene que es a la familia, a los amigos, a los compañeros de trabajo y a los jefes a quienes los pacientes con lepra tienden a ocultar, no sólo el nombre, sino la afección misma. Con ello se ratifica lo señalado por Goffman.

"él (el jefe) no sabía nada, porque yo no le había dicho nada”. (Lilia)

“no, no quiero (decirlo)... porque en el medio donde... yo me desenvuelvo, no es quedarse callado, es hacer comentarios; ahorita yo no confío en nadie". (Janeth)

El encubrimiento se produce hasta en el caso de aquellos sujetos cuyo atributo es evidente - tal como ocurre en uno de los entrevistados con grado II de incapacidad (desacreditado) -, que aún cuando sus deformidades queden al descubierto, enmascara su condición de leproso.

"Yo trataba más bien de ocultar delante de todos que yo tenía la mano derecha fregá (dañada)...”. (Martín)

\section{Aislamiento}

Otro tipo de situación se presenta cuando en el intercambio social el paciente con lepra se aisla, razón que le hace volverse desconfiado, depresivo, hostil, ansioso y aturdido. Según Goffman, éstas son características resaltantes, en un sujeto que se excluye por voluntad propia.

De los pacientes entrevistados, sólo dos refieren una marcada tendencia hacia la autoexclusión. El primero sólo al inicio de la enfermedad, pero la segunda se ha mantenido aislada desde el principio:
"Yo quise estar solo, o sea, un tiempo, para meditar qué era to que yo iba hacer conmigo y así estuve un tiempo”. (Martín)

“... No quiero que nadie se entere... de hecho me he apartado de mis amistades... yo me encerré aquí en mi casa... a mis amigos si los trato, pero no igual que antes... entonces no quiero que nadie me hable... ni me pregunten qué es to que me pasa... yo no quiero hablar, o sea, eso es mío, de más nadie”. (Janeth)

\section{DISCUSION}

1. Dado que el estereotipo que se tiene acerca de las personas con lepra constituye la forma más simple de expresión de la representación social al respecto, resulta significativa la imagen que el propio enfermo proyecta de sí, en cuanto ésta condensa ciertos rasgos o características configuradoras de la estigmatización.

Así vemos que el enfermo se representa al leproso como una persona que pierde piel, o como una persona llagosa. Tal imagen evoca, sin lugar a dudas, a un tipo de leproso en particular, a aquel a quien la enfermedad le ocasiona lesiones corporales que hacen evidente el padecimiento. El predominio de esta imagen en el propio afectado es revelador de que éste tiende a reducir su representación social a una categoría de sujetos - los deformes o mutilados -, que ancestralmente se ha configurado como la portadora del estigma. Tal categoría se identifica con el tipo de estigmatizado llamado por Goffman el desacreditado. Sin embargo, junto a esa imagen, parece coexistir una más general y menos estigmatizante, donde aparece una persona que tieue la piel manchada y que puede perder piel, o que es débil físicamente. Ello explica el elevado valor del índice de estereótipización (68\%).

2. Dentro de los factores que el propio enfermo asume como lo que le impide una integración social, se encuentra 
el temor a ser rechazado por las otras - personas, por los amigos, por la pareja. También es intenso su temor a ser expulsado del trabajo, así como el ser percibido como una persona diferente. Así pues, el temor a ser rechazado o excluido representa para el enfermo el principal y más grave problema que debe enfrentar y resolver para poder desarrollar una vida social, laboral y familiar armoniosa. Otro factor de la inhabilitación es el ocultamiento, que constituye una tendencia generalizada en el paciente, obligándolo o presionándolo a adoptar con frecuencia comportamientos de enmascaramiento y/o encubrimiento. Ello es así por la intensidad de su temor a ser rechazado.

Sin embargo, la percepción de la inhabilitación social que tiene el enfermo (dimensión subjetiva) contrasta con su capacidad de integración y con la que se le atribuye al leproso. Así to describe una persona que puede trabajar, si no tiene lesiones graves, y que puede realizar su trabajo con un rendimiento similar al que tenía antes de aparecerla enfermedad. Por otro lado, no sólo se percibe como una persona que no es rechazada por su familia, sino que considera tener una importante proximidad con ella. Tal contraste sin embargo, no debe llevar a perder la perspectiva en relación a la inhabilitación social ya que, tal como to expresa el coeficiente calculado (67\%), ésta opera en todos los leprosos, independientemente de la inexistencia de deformidades y de la actitud favorable de algunos a participar activamente en la vida social.

\section{RESUMEN}

ROMERO-SALAZAR, A.; PARRA, M. C.; MOYA-HERNANDEZ, C.; RUJANO, R. \& SALAS, J. El Estigma en la Representación Social de la Lepra. Cad. Saúde Públ., Rio de Janeiro, 11 (4): 535-542, oct/dec, 1995.

La representación social de la lepra en el paciente activo bajo control se constituye de elementos provenientes del discurso médico y del discurso que, en torno a la afección, circula en la sociedad. Tal sincretismo se expresa, en cada contexto, de una manera específica, sobre los componentes de la representación nociones, creencias, actitudes y conductas haciendo de ella una realidad compleja. En razón del peso que tienen las creencias, cuya profundidad y arraigo están vinculadas a la antigüedad de la enfermedad y a las prácticas confinatorias vigentes hasta épocas recientes, sería comprensible el predominio de los elementos ligados al sentido común o saber popular. No obstante, siendo la representación una construcción colectiva, sus rasgos fundamentales estarían definidos por las específicas características del grupo. Al respecto, los principales hallazgos de la investigación se refieren, primero, al escaso conocimiento de los pacientes activos en el Servicio de Dermatología Sanitaria de Maracaibo (Venezuela) en torno a la enfermedad y a la existencia de creencias y actitudes inadecuadas para los programas de control: segundo, al predominio, en la dimensión cognoscitiva de la representación, de nociones y creencias en relación al papel de la suerte o del destino, de la promiscuidad sexual o de la herencia en la transmisión; tercero, a la tendencia a la participación del paciente en la reproducción de un estereotipo descalificador y al ocultamiento, aunado a un exagerado miedo al rechazo.

Palabras Clave: Lepra; Representación Social; Estigma 


\section{REFERENCIAS BIBLIOGRAFICAS}

CLARO, B. L., 1995. Hanseníase Representações sobre a Doença. Rio de Janeiro: Editora Fiocruz.

DE ROJAS, V., 1989. Nivel de conocimientos de los enfermos de lepra de ciudad de la Habana en relación con su padecimiento. Revista Cubana de Medicina Tropical, 41: 64-75.

FASSIN, D., 1990. Influence of social perceptions of leprosy and leprosy patients on public health programs. International Journal of Leprosy, 58: 111-114.

GOFFMAN, E., 1961. Internados. Buenos Aires: Amorrortu. 1970. Estigma. Buenos Aires: Amorrortu.

HASSELBLAD, O. W., 1975. Aspectos psicosociales de la lepra. Boletín de la Oficina Sanitaria Panamericana, 78: 422-429.
KAUFFMAN, A. E., 1985. La Lepra y sus Imágenes. Enfermedad, Estigma y Muerte Social. Madrid: Ministerio de Trabajo y Seguridad Social.

POIRIER, J.; CLAPIER-VALLEDON, R. \& RAYBAUT, C., 1983. Les Recits de Vie: Théorie et Pratigue. Paris: Presses Universitaires de France.

RODRIGUEZ, J. C., 1983. Tabu do Corpo. Rio de Janeiro: Achiamé.

ROMERO-SALAZAR, A., 1990. Diversidad o especificidad del análisis sociológico. Revista Contextos (Maracaibo), pp. 40-43.

SANCHES, O. \& MINAYO, M. C. S., 1993. Quantitativo-qualitativo: oposição ou complementaridade? Cadernos de Saúde Pública, 9: 239-248.

VALENCIA, L. B., 1989. Social science research on social dimensions of leprosy: Where are we going from here? International Journal of Leprosy, 57: 847-863. 